\title{
Older adults and withdrawal from benzodiazepine hypnotics in general practice: effects on cognitive function, sleep, mood and quality of life
}

\author{
H. V. CURRAN, ${ }^{1}$ R. COLLINS, S. FLETCHER, S. C. Y. KEE, \\ B. WOODS AND S. ILIFFE
}

\begin{abstract}
From the Psychopharmacology Research Unit, Clinical Health Psychology, University College London and Centre for Ageing Population Studies, Department of Primary Care and Population Sciences, Royal Free and University College London Medical School, London; and School of Psychology, University of Wales, Bangor
\end{abstract}

\begin{abstract}
Background. Older adults are the main recipients of repeat prescriptions for benzodiazepine (BZD) hypnotics. BZDs can impair cognitive function and may not aid sleep when taken continuously for years. This study therefore aimed to determine if withdrawing from BZDs leads to changes in patients' cognitive function, quality of life, mood and sleep.
\end{abstract}

Method. One hundred and ninety-two long-term users of BZD hypnotics, aged $\geqslant 65$ years, were identified in 25 general practices. One hundred and four who wished to withdraw were randomly allocated to one of two groups under double-blind, placebo controlled conditions: group A's BZD dose was tapered from week 1 of the trial; group B were given their usual dose for 12 weeks and then it was tapered. An additional group (C) of 35 patients who did not wish to withdraw from BZDs participated as 'continuers'. All patients were assessed at 0,12 and 24 weeks and $50 \%$ were reassessed at 52 weeks.

Results. Sixty per cent of patients had taken BZDs continuously for $>10$ years; $27 \%$ for $>20$ years. Of all patients beginning the trial, $80 \%$ had successfully withdrawn 6 months later. There was little difference between groups A and B, but these groups differed from continuers $(\mathrm{C})$ in that the performance of the withdrawers on several cognitive/psychomotor tasks showed relative improvements at 24 or 52 weeks. Withdrawers and continuers did not differ in sleep or BZD withdrawal symptoms.

Conclusions. These results have clear implications for clinical practice. Withdrawal from BZDs produces some subtle cognitive advantages for older people, yet little in the way of withdrawal symptoms or emergent sleep difficulties. These findings also suggest that, taken long-term, BZDs do not aid sleep.

\section{INTRODUCTION}

Long-term use of benzodiazepine (BZD) hypnotics is significantly higher in older adults than younger people throughout North America, Australia and Europe (Barbui et al. 1998; Egan et al. 2000; Colenda et al. 2002). It has been

\footnotetext{
1 Address for correspondence: Professor H. Valerie Curran, Psychopharmacology Research Unit, Clinical Health Psychology, University College London, Gower Street, London WC1 6BT.
}

estimated that around $15 \%$ of over 65 -year-olds regularly take sleeping pills and, in the UK, older adults receive $80 \%$ of all the prescriptions written for BZD hypnotics (Morgan \& Clarke, 1995; Jorm et al. 2000). Although BZDs are not nowadays recommended for long-term use $(>4$ weeks), hypnotics are commonly given to over 65-year-olds in repeat prescriptions.

It is not clear whether BZDs taken over prolonged periods remain effective in actively 
promoting sleep as opposed to preventing withdrawal symptoms and rebound insomnia (Lader, 1992). A review of drug use by older adults concluded: 'After tobacco and alcohol, benzodiazepine consumption is associated with the greatest risk of abuse and dependence in the elderly' (Ticehurst, 1995).

Older adults are more susceptible to the effects of BZDs and other psychotropics because agerelated changes in pharmacokinetic processes (particularly distribution, metabolism and clearance) can greatly prolong the effects of these drugs (Greenblatt et al. 1991). Older people often take a combination of drugs, including centrally-acting ones, which can compound side-effects. Cognitive effects of benzodiazepines are of particular concern for older adults whose everyday function may be especially vulnerable to memory failures. Age-related cognitive decline may be exacerbated by additional druginduced impairment with substantial repercussions for older people's daily functioning, including in some cases confusional states and 'pseudo-dementia' (Shorr \& Robin, 1994). Indeed, it has been estimated that about $10 \%$ of older adults referred to memory clinics display cognitive impairments that are drug-related, often due to benzodiazepines (Gray et al. 1999). There is also a clear association between the use of BZDs by elderly people and increased risk of falls and fractures, and of road traffic accidents (Campbell, 1991; Woods et al. 1992; Barbone et al. 1998).

Although there has been a reduction in scripts for BZDs since the mid-eighties, this reflects a substantial decrease in BZD anxiolytics but relatively little change in BZD hypnotics especially among older adults (Rumble \& Morgan, 1994; Woods \& Winger, 1995). A large-scale audit of BZD prescribing and withdrawal in general practices found that patients over 65 are significantly less likely to stop BZDs than younger patients (Holden et al. 1994). Any effort to reduce use of BZDs should therefore focus on the main users, older adults.

More than 100 studies have documented cognitive impairments produced by BZDs but very few focus on older people. This literature is consistent in showing marked impairments in memory and concentration following single daytime doses of a BZD (Curran, 1991, 2000; Woods et al. 1992). Most studies that have involved people over 65 years-of-age have looked at effects of a single dose of BZD and therefore have limited clinical relevance. These have found either similar or greater levels of impairment that persist significantly longer in older compared to younger people (Pomara et al. 1985, 1998; Hinrichs \& Ghoneim, 1987; Nikaido et al. 1990; Greenblatt et al. 1991). Residual effects on daytime performance following single or several night-time doses have also been documented in older people (Morgan, 1994). Despite the widespread long-term use of these drugs, we still, as Griffiths (1995) laments, know very little about their long-term effects in any age group. The only randomized controlled study of cognitive effects in patients showed very significant memory impairments following 2 months treatment with a BZD compared with placebo (Curran et al. 1994). A cross-sectional study comparing a group of long-term ( $>6$ months) BZD users with other groups found BZD consumption over years correlated with impairments in attentional and visuospatial functions (Golombok et al. 1988).

If withdrawal from BZD hypnotics resulted in a reversal of drug-induced impairment (i.e. improved cognitive and psychomotor function) then there would be clear benefits for older people. Older people often complain of memory problems and may see these as due to age rather than additional drug induced problems. Improved cognitive and psychomotor function may enhance daily functioning and quality of life. The scope for such an effect is indicated by Salzman et al. (1992). They assessed memory performance of 25 people (average age 86 years), all residents in a nursing home who had taken BZDs for several months or years. Thirteen agreed to withdraw from BZDs and 12 wished to continue their BZD use. Initially, there were no differences between the two groups, but 2-3 weeks after the first group had stopped taking BZDs, they performed significantly better on tests of working and episodic memory than those who had continued medication. Care staff rated the discontinuers as more alert and less forgetful in their daily functioning than the continuers. This study was not conducted doubleblind, it used limited measures and sample sizes were small. Nevertheless, the positive effects observed a few weeks after withdrawal were encouraging. 
The present study was designed to investigate the effects of withdrawal from BZD hypnotics in a larger, primary care sample of older adults. Using double-blind procedures, it assessed patients a few weeks after withdrawal and then again at 6 and 12 months. It aimed to determine both the benefits and drawbacks of withdrawing from BZDs on cognitive function within the broader, clinical picture of withdrawal effects on the older person's health-related quality of life, sleep, mood and bodily symptoms.

\section{METHOD}

\section{Participants and design}

Twenty-five general practices were recruited in inner city and suburban London and in rural areas from the teaching and research network of the Royal Free and UCL Medical School. All patients who were $\geqslant 65$ years-of-age and taking BZD hypnotics on a repeated, daily basis for at least 6 months were identified through an audit of each of the 25 practices' computer records. Researchers and general practitioners (GPs) excluded those patients with dementia or other organic states associated with cognitive dysfunction; severe deafness or severe visual impairment; current major psychiatric disorders; histories of seizures; those receiving terminal care. GPs could also exclude any patient for whom they felt discontinuation of BZD hypnotics was clinically inappropriate. The remaining patients were sent a letter signed by their GP inviting them to attend an appointment with a research psychologist to discuss their sleep problems and their use of sleeping tablets. Each patient was interviewed individually and their attitudes and beliefs about BZDs and sleep were explored in a semi-structured interview (Iliffe et al. 2003). At the end of the interview, each patient was invited to participate in a withdrawal programme.

Patients wishing to discontinue their sleeping tablets were randomly allocated to one of two treatment groups (A or B) in a double-blind, independent group design. Following baseline assessment, patients in group A had their dose of BZD gradually tapered over the first 8 or 9 weeks and then remained on placebo through to week 24; patients in group B continued taking their normal BZD for the next 3 months and then received the same intervention as group A.
Patients were assessed at three time points: week 0 (baseline), 12 and 24. A proportion of patients were followed up and re-assessed at week 52 . Test versions were counterbalanced across participants and design.

The third group of patients (group C) who wished to continue their normal use of sleeping pills was assessed on the same tasks at the same time points. Random allocation to this group was precluded for ethical reasons. The design of the study is summarized in Fig. 1. The study was approved by all relevant local ethical committees and all participants gave written, informed consent.

\section{Medication}

For patients in groups A and B, all drugs were formulated in identical opaque capsules and packed with lactose placebo to appear the same throughout the trial. A dose titration regime was devised to minimise the risk of withdrawal symptoms and this was done according to each patient's original dose and particular BZD. For example, for patients in group A, $10 \mathrm{mg}$ of temazepam was reduced by $2.5 \mathrm{mg}$ every 2 weeks according to the following schedule: week 1 (10 mg); weeks 2 and $3(7.5 \mathrm{mg})$; weeks 4 and 5 (5 mg); weeks 6 and 7 (2.5 mg); week 8 onwards (0 $\mathrm{mg}$ i.e. placebo only). For patients in group $\mathrm{B}$, the schedule was parallel with dose reduction beginning at week 13 . The schedule for $5 \mathrm{mg}$ nitrazepam was: week $1(5 \mathrm{mg})$, weeks 2 to 5 $(2.5 \mathrm{mg})$, weeks 6 to $12(0 \mathrm{mg})$. The schedule was adjusted for larger doses. For example, the schedule for $20 \mathrm{mg}$ temazepam was: week 1 $(20 \mathrm{mg})$; week 2 and $3(15 \mathrm{mg})$; week 4 and 5 $(10 \mathrm{mg})$; week 6 and $7(5 \mathrm{mg})$; week 8 and 9 $(2.5 \mathrm{mg})$; week 10 onwards $(0 \mathrm{mg})$.

Tablet bottles were numbered consecutively from 1 to 24 and were given in monthly supplies to the patient or left at the GP surgery for the patient to collect. Tablet bottles were returned after use and pill counts made as an adherence check. As an additional check to confirm BZD withdrawal, urine samples were collected from patients in groups A and B at 52 weeks (as well as at baseline) for analysis of BZDs.

\section{Psychological support}

Researchers trained in giving psychological support saw each patient at initial recruitment 


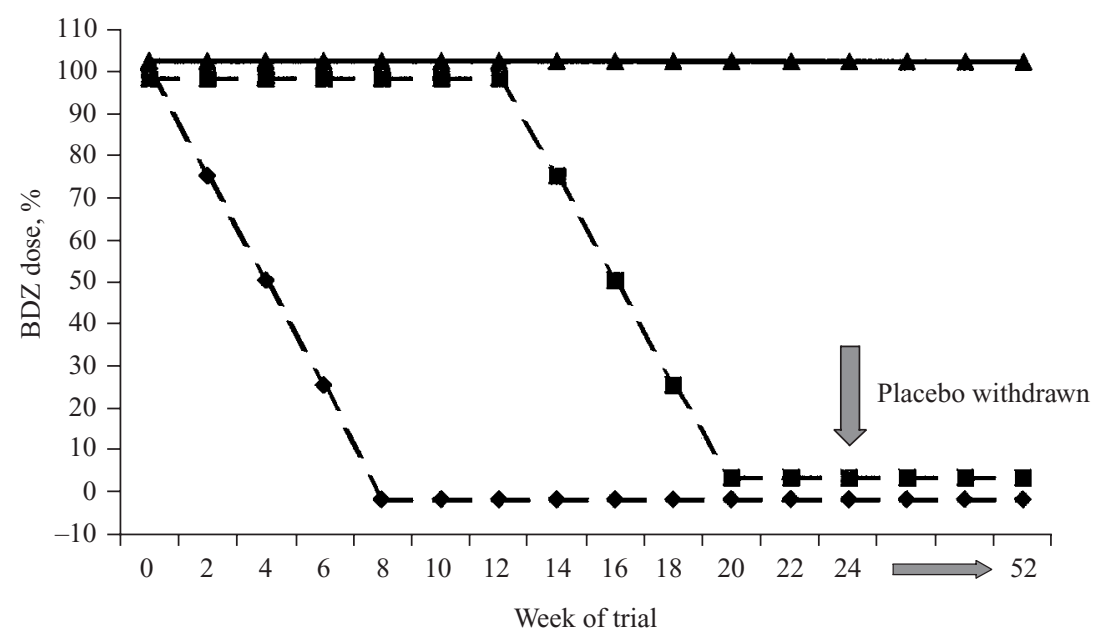

FIG. 1. Group design of the study (see text for dose titration schedules) $\boldsymbol{\Lambda}$, continuers (group C)).

and at the four assessment times. Researchers were blind to group allocation. A pamphlet on sleep and sleep hygiene was given to each patient and telephone support was also available to patients when needed. These measures aimed to both maximize the success rates of BZD withdrawal and to minimize any demands on GPs' time.

\section{Assessments}

Assessments were selected according to the following criteria: ( $i$ ) to sample the range of memory, attentional and psychomotor functions sensitive to impairment by BZDs; (ii) to be clinically relevant in that most of the tests sample 'daily-life' cognitive requirements; (iii) to be appropriate to and not unduly fatiguing for older adults; (iv) to monitor sleep, mood, bodily symptoms and possible withdrawal symptoms; and $(v)$ to monitor health-related quality of life. The whole battery took approximately $50 \mathrm{~min}$ to complete.

\section{Cognitive assessments}

Spot the Word task (Baddeley et al. 1992)

This assesses pre-morbid intellectual function and correlates well $(0.87)$ with the widely used National Adult Reading Test (NART). It is however, less anxiety inducing than the NART. It was administered once at the beginning of the trial. The tests listed below were administered at each assessment with test versions being counterbalanced across participants and design.

Speed of Comprehension Test (Baddeley et al. 1992)

This taps semantic processing speed. Participants were presented with a list of simple statements (e.g. 'ants are living creatures', 'vans grow in gardens') and given 2 min to mark as many sentences as possible as true or false.

\section{Prose recall}

This subtest from the Rivermead Behavioural Memory Test (Wilson et al. 1985) taps episodic memory. Participants listened to a tape-recorded passage (like a 'news bulletin' on the radio) and were asked to recall it aloud immediately afterwards and again after a delay during which the other tests were completed.

\section{Map location task}

This is a subtest from the Tests of Everyday Attention (Robertson et al. 1996) that taps visuospatial search abilities. Participants were shown a target symbol on a large, coloured map and then given 2 min to locate as many instances of the specified symbol as possible. The number of symbols found is recorded separately for each of the $2 \mathrm{~min}$. 


\section{Digit span}

Stimuli were digits 1-9 read aloud in increasing strings of random sequences at the rate of 1 digit per second. This subtest of the WAIS (Wechsler, 1955) taps working memory and attention. Score was the total of span forwards and backwards.

\section{Speed of information processing task}

A target number is randomly selected and constantly displayed to the right of notebook computer screen (Wesnes et al. 2000). A series of digits is then presented in the centre of the screen at the rate of 120 per min and the volunteer is required to press the 'YES' button on a response box as quickly as possible every time the digit in the series matches the target digit. Reaction times and correct/incorrect responses are recorded automatically. The task lasts for 3 min and taps speed of information processing, attention and working memory.

\section{Assessments of alertness and psychomotor speed}

\section{Simple reaction time}

The simple reaction time to 24 stimuli presented at random intervals on a screen of a notebook computer was recorded. The participant pressed a large space bar as quickly as possible when a stimulus (a flower) appeared on the screen.

\section{Tapping speed}

The participant was asked to press the space bar of a computer key board with their preferred hand as quickly as possible for $1 \mathrm{~min}$. This task measures the individual's capacity to rapidly execute simple repetitive manual operations. The number of key depressions made in $1 \mathrm{~min}$ is used an index of psychomotor speed.

\section{Health-related quality of life}

SF-36 (Medical Outcomes Study Short-form 36)

This was used to measure health related quality of life. The reliability and validity of this short scale has been demonstrated with older adults (Lyons et al. 1994; Andersen et al. 1995; Hayes et al. 1995). The SF-36 (with scaled responses to questions) is more sensitive than the Nottingham health profile (NHP) to low levels of disability (Bowling, 1991). An error in copying resulted in incomplete scores on one of the eight factors (general health) and data on this factor was not valid. The SF-36 was completed on behalf of the patient by the researcher so as to overcome the problems of self-administration of this scale with older adults.

\section{BZD withdrawal symptoms, other somatic symptoms and mood}

The Benzodiazepine Withdrawal Symptom Questionnaire (Tyrer et al. 1990) is a set of visual analogue scales (VAS) concerning BZD withdrawal symptoms (e.g. perceptual sensitivity) and participants rated the extent they had experienced the symptoms in the previous $24 \mathrm{~h}$. Mean ratings across all scales were calculated. Patients also rated somatic symptoms on a VAS bodily symptoms scale and their mood at the time of each assessment on the mood rating scale (Bond $\&$ Lader, 1974). This yields three mood factors: alertness, contentedness and calmness. The Geriatric Depression Scale (short form) (D'Ath et al. 1994) is a 15-item scale that has been validated with older adults and it was used to assess depression.

\section{Sleep}

Patients withdrawing from their sleeping tablets completed a sleep diary based on Morgan \& Gledhill (1991) for each of 7 days beginning at each assessment point. Average (mode) ratings of sleep over the 7 days were calculated. In addition, VASs anchored 'no problems sleeping' and 'severe problems sleeping', as well as 'no dreams' and 'very vivid dreams' were also completed by all patients at each assessment time.

\section{Statistical analyses}

There were two main stages, first comparing groups A and B and secondly comparing group $C$ (continuers) with the combined group of withdrawers $(\mathrm{A}+\mathrm{B})$. To compare groups $\mathrm{A}$ and $B$, repeated measures ANOVAs were carried out on data for patients who had completed all assessments to 24 weeks (the placebo controlled trial period) and then for those completing the 52 week follow-up (as compared with baseline). Age was used as a covariate when significant group or time differences emerged; pre-morbid IQ (Spot the Word) was also covaried from performance test data. Repeated measures ANOVAs were used to compare group $\mathrm{C}$ with 
Table 1. Demographics of patients in each group: numbers (N), mean (S.D.) age, \% female, pre-morbid function (Spot the Word), current diazepam-equivalent dose of BZD taken and years of continuous BZD use

\begin{tabular}{llll}
\hline \hline & Group A & Group B & Group C \\
\hline$N$ & 55 & 49 & 34 \\
Age (years) & $76 \cdot 0(6 \cdot 8)$ & $77 \cdot 7(6 \cdot 7)$ & $77 \cdot 2(7 \cdot 5)$ \\
Female, \% & 73 & 65 & 77 \\
Spot the Word (score) & $48 \cdot 3(8 \cdot 4)$ & $48 \cdot 2(8 \cdot 9)$ & $49 \cdot 2(8 \cdot 4)$ \\
Current BZD: \% temazepam/\% nitrazepam & $69 / 24$ & $67 / 29$ & $48 / 47$ \\
Current dose (DZ equivalent mg) & $11 \cdot 7(4 \cdot 7)$ & $11 \cdot 6(4 \cdot 8)$ & $11 \cdot 9(4 \cdot 9)$ \\
Years of BZD use & $14 \cdot 1(8 \cdot 1)$ & $13 \cdot 0(8 \cdot 1)$ & $15 \cdot 1(7 \cdot 5)$ \\
\hline \hline
\end{tabular}

combined groups $\mathrm{A}$ and $\mathrm{B}$ at the same time points. An intention to treat analysis over all four assessment periods was also performed. Chi-square tests were used for categorical data.

\section{RESULTS}

\section{Demographics}

Of 192 patients who were interviewed and complied with relevant inclusion/exclusion criteria, 138 agreed to participate in the study. One hundred and four patients chose to participate in the withdrawal trial and a further 34 patients chose to take part in the study although they did not wish to change their use of BZD hypnotics (group C).

Patients ranged in age from 65 to 93 (mean: $77 \pm 6.9$ ) years and there was no difference in age between patients in groups A, B and C $(F<1 \cdot 0)$ (Table 1). Seventy-one per cent of the total sample of patients was female and there were no significant group differences in numbers of males and females. Pre-morbid function (Spot the Word scores) showed no differences between the three groups $(F<0 \cdot 2)$.

\section{BZD hypnotics}

The majority of patients were taking temazepam (mostly $10 \mathrm{mg}$ ), about a third were taking nitrazepam (mostly $5 \mathrm{mg}$ ) and the remainder were taking loprazolam (Table 1). Diazepam equivalent doses were calculated $(10 \mathrm{mg}$ diazepam $=$ $10 \mathrm{mg}$ temazepam $=5 \mathrm{mg}$ nitrazepam $=1 \mathrm{mg}$ loprazolam) and there were no group differences in dose $(F<0 \cdot 05)$. About $60 \%$ of patients had been taking BZD hypnotics for $>10$ years, $27 \%$ had taken them for $>20$ years and there were no significant group differences in length of BZD treatment. A few patients took other psychotropic mediation besides BZD hypnotics (one
Table 2. Number of patients in each group at each 0,12 and 24 week assessment point and number followed up at week 52

\begin{tabular}{rrrcc}
\hline \hline & Baseline & 12 weeks & 24 weeks & $(52$ weeks $)$ \\
\hline Group A & 55 & 48 & 45 & $(30)$ \\
Group B & 49 & 43 & 38 & $(22)$ \\
Group C & 34 & 27 & 21 & $(15)$ \\
Total & 138 & 118 & 104 & $(67)$ \\
\hline \hline
\end{tabular}

patient in group A and four patients in group C took other BZDs as daytime anxiolytics; four patients in group A, two in group B and three in group $\mathrm{C}$ took antidepressants).

\section{Patients successfully withdrawing from BZDs}

Of the 104 patients who entered the trial, $80 \%$ successfully withdrew from their BZDs and were re-tested at 24 weeks. The drop out rate from the trial was surprisingly low (Table 2). Figures in Table 2 for week 52 are given in parentheses because those entering late in the trial period $(N=24$, including all patients taking loprazolam) could not be assessed beyond 24 weeks as the project had ended.

From the 104 patients who agreed to discontinue their BZD hypnotics, three changed their minds at the beginning of the trial, two (both from group B) were unhappy with the assessments and another patient (B) took one capsule before withdrawing from the trial altogether. Between 0 and 12 weeks, reasons for drop out were that two patients (one in group A, one in B) suffered major illnesses, one patient (B) died, one patient's (A) spouse died, one patient (A) had problems swallowing the capsules and another (A) said the capsules made her sick. A further four patients (two from group A and two from group B) gave no reason for not 
Table 3. Group means (S.D.) on cognitive and psychomotor tests for all patients tested at weeks 0,12 and 24; the last two columns present baseline (week 0) and week 52 data for the subgroup who were assessed at the week 52 follow-up

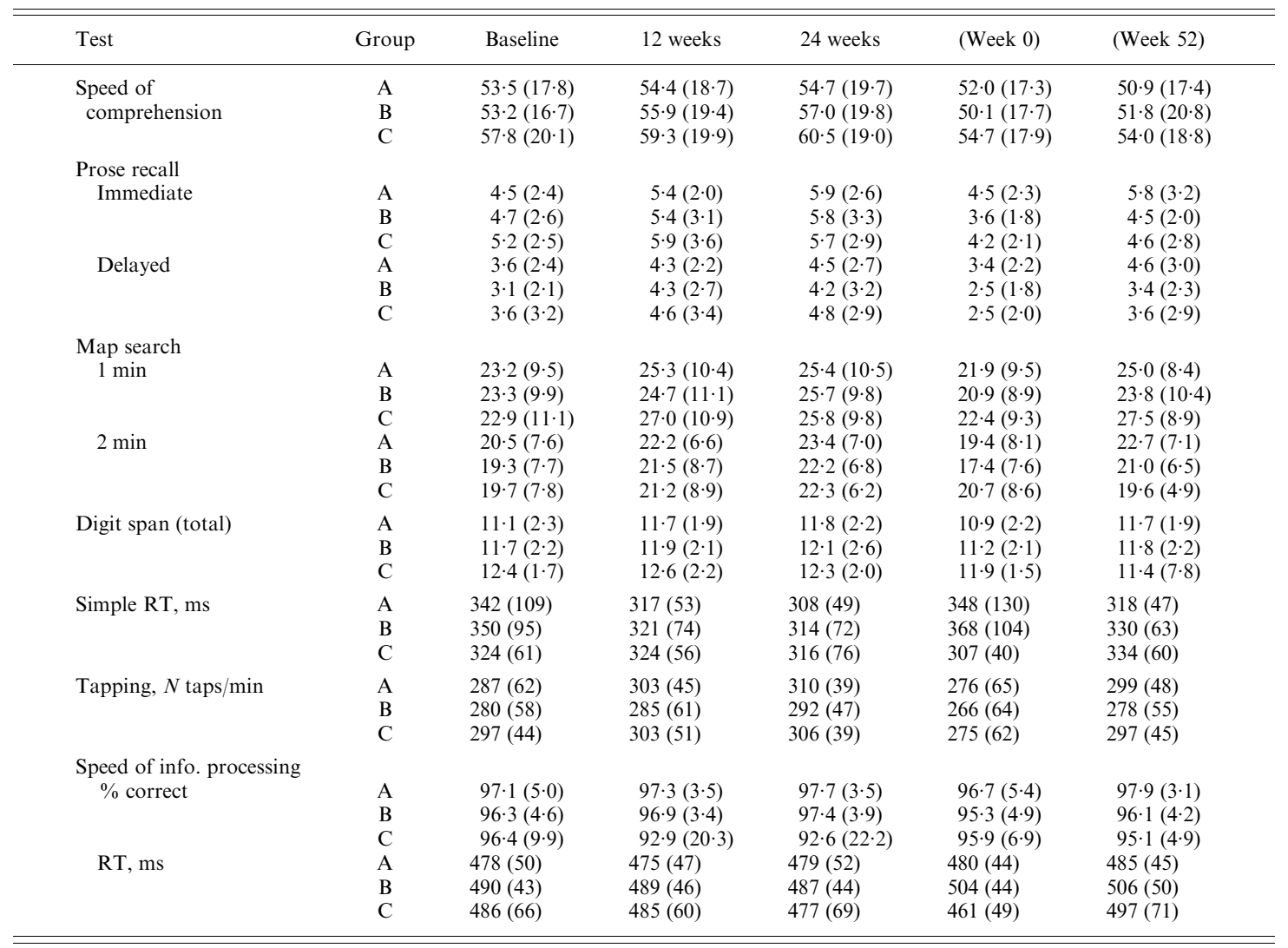

wishing to continue the trial. Between 12 and 24 weeks, a further three patients stopped the programme: one (A) had a major illness; one (A) was admitted into hospital and another patient (B) died. Five patients chose to withdraw from the trial without stating a reason (one from group A and four from group B). It can be seen from Table 2 that patients were no more likely to drop out of the trial when they were withdrawing from BZDs than when they were taking their usual dose.

\section{Urine}

Urine samples were obtained from 27 patients in groups $\mathrm{A}$ and $\mathrm{B}$ at both baseline and one year follow-up. All screened positive for BZDs at week 0 and negative at week 52 , confirming withdrawal.

\section{Effects of withdrawal on cognitive and psychomotor function}

\section{Comparison of group $A$ with group $B$}

No significant differences emerged in comparing these two intervention groups (see Table 3). On most measures, there was a significant effect of week that was no longer significant when age was covaried (immediate and delayed recall of prose; map search min 1 and 2; total digit span; simple reaction time; tapping speed). On the other measures (speed of comprehension, accuracy and reaction time in speed of information processing) there were no significant effects of week but again, age was a significant covariate. Pre-morbid IQ was a significant covariate on speed of comprehension. Analyses on an intent to treat basis showed the same effects. 
Comparison of withdrawers (combined groups $A$ and $B)$ with continuers $(C)$

Comparisons of groups over the first 24 weeks of the trial showed a significant group $\times$ week interaction for accuracy in speed of information processing $\left(F_{2,96}=6 \cdot 31, P=0.003\right)$ and a main effect of group $\left(F_{1,97}=4 \cdot 09, \quad P<0 \cdot 05\right)$. Age $\left(F_{1,94}=4.66, \quad P<0.05\right)$ and pre-morbid IQ $\left(F_{1,94}=5 \cdot 31, P<0.05\right)$ were significant covariates, and when these were covaried the interaction between week and group $\left(F_{2,93}=5.98\right.$, $P<0.005)$ and the group main effect remained $\left(F_{1,94}=4 \cdot 83, P<0 \cdot 05\right)$. As seen in Table 3, withdrawers' performance slightly improved over time compared with a declining performance by those who continued medication.

Other measures over weeks $0,12,24$ showed a significant effect of week that was no longer significant when age and pre-morbid IQ were covaried (immediate and delayed recall of prose; map search min 1 and 2; total digit span; simple reaction time; tapping speed). The speed of comprehension and simple reaction time tasks showed no significant effects (apart from age and pre-morbid IQ being significant covariates).

Comparing week 52 performance with baselines, there were significant group $\times$ week interactions on four variables (with age and pre-morbid IQ covaried): map search min 2 $\left(F_{1,60}=4 \cdot 26, P<0 \cdot 05\right)$, reaction time in speed of information processing $\left(F_{1,59}=11 \cdot 97, P<0.001\right)$, total digit span $\left(F_{1,60}=8.59, P<0.005\right)$; simple reaction time $\left(F_{1,60}=5 \cdot 61, P<0 \cdot 05\right)$. On these measures, the continuers (Group C) had higher week 0 performance than withdrawers. However, withdrawers improved between 0 and 52 weeks whereas group $\mathrm{C}$ showed a relative decline. The map search task showed the most marked improvement of withdrawers whose performance at week 52 was $22 \%$ better than their week 0 performance (see Fig. 2, which depicts means for those patients assessed at both time points).

\section{Effects of withdrawal on mood, health-related quality of life and somatic symptoms}

\section{Comparison of group $A$ with group $B$}

(Tables 4 and 5) Few significant differences emerged in comparing these two intervention groups. On several measures, there was a significant effect of week that was no longer

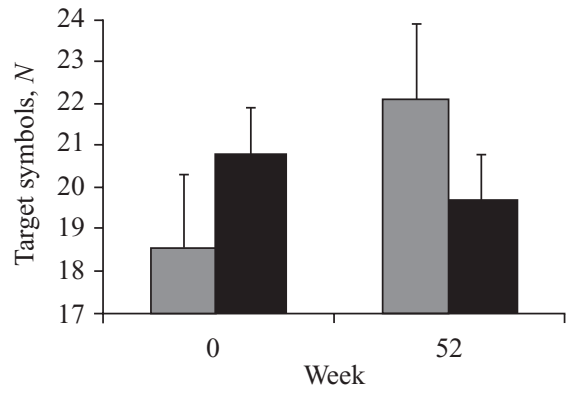

Fig. 2. Mean scores of withdrawers ( $\square$, groups $\mathrm{A}$ and B) and continuers $(\boldsymbol{\square}, \mathrm{C})$ on Map Search (2 min) at weeks 0 and 52 .

significant when age was covaried (BZD withdrawal scale; geriatric depression scale; ratings of impaired concentration). There was a main effect of group on the social functioning scale of the SF-36, reflecting higher scores by group B than A throughout the trial. There was also a main effect of group on the physical role scale of the SF-36, reflecting higher scores by group B than A throughout the trial. Analyses on an intent to treat basis showed the same effects.

\section{Comparison of withdrawers (combined groups $A$ and $B$ ) with continuers $(C)$}

The geriatric depression scale (GDS) (Table 4) showed a main effect of week that was no longer significant when age was covaried. Two individual body symptoms scales showed group $\times$ week interactions across weeks $0-24$ (Table 4). Ratings of anxiety showed a marked increase at week 24 in group $\mathrm{C}$ compared with the withdrawal groups $\left(F_{2,101}=4.66, P<0.05\right)$; the same pattern emerged for ratings of impaired concentration $\left(F_{2,101}=4 \cdot 12, P<0.05\right)$. Two individual scales of body symptoms showed group differences (main effects): irritability and lack of energy whereby group $\mathrm{C}$ scored somewhat higher than groups $\mathrm{A} / \mathrm{B}$ throughout the trial. No significant effects emerged on other body symptoms scales, on mood factors of the MRS or on the BWSQ.

There were group differences (main effects) on some subscales of the SF-36 but no interactions (Table 5). The withdrawal groups had higher scores (reflecting increased health) than group $\mathrm{C}$ on 'physical role' across weeks $0-24\left(F_{1,98}=\right.$ $10 \cdot 17, P<0.005)$, and on 'social functioning' weeks $0-24\left(F_{1,94}=5.40, P<0.05\right)$ and week $52\left(F_{1,61}=6.25, P<0.015\right)$. On the subscale 
Table 4. Group means (S.D.) on the benzodiazepine withdrawal scale (BWSQ), Geriatric Depression Scale (GDS), mood factors and on visual analogue scales ( $\mathrm{mm}$ ) showing significant effects for all patients tested at weeks 0,12 and 24 (week 52 data are for the subgroup assessed at follow-up)

\begin{tabular}{|c|c|c|c|c|c|}
\hline & Group & Baseline & 12 weeks & 24 weeks & (Week 52) \\
\hline \multirow[t]{3}{*}{ BWSQ } & A & $34 \cdot 4(18 \cdot 7)$ & $34 \cdot 8(20 \cdot 4)$ & $29 \cdot 3(15 \cdot 7)$ & $24 \cdot 8(12 \cdot 5)$ \\
\hline & $\mathrm{B}$ & $34 \cdot 5(13 \cdot 7)$ & $32 \cdot 7(16 \cdot 5)$ & $27 \cdot 3(12 \cdot 4)$ & $24 \cdot 7(8 \cdot 3)$ \\
\hline & $\mathrm{C}$ & $33 \cdot 4(11 \cdot 1)$ & $29 \cdot 8(10 \cdot 6)$ & $32 \cdot 8(13 \cdot 4)$ & $31 \cdot 6(9 \cdot 5)$ \\
\hline \multirow[t]{3}{*}{ GDS } & A & $3 \cdot 9(3 \cdot 0)$ & $3 \cdot 0(2 \cdot 9)$ & $2 \cdot 9(3 \cdot 0)$ & $1 \cdot 7(2 \cdot 5)$ \\
\hline & B & $3 \cdot 5(2 \cdot 8)$ & $2 \cdot 7(2 \cdot 7)$ & $1.9(2 \cdot 1)$ & $1 \cdot 3(2 \cdot 1)$ \\
\hline & $\mathrm{C}$ & $3 \cdot 4(2 \cdot 8)$ & $2 \cdot 7(2 \cdot 7)$ & $2.9(3.6)$ & $1 \cdot 7(2 \cdot 8)$ \\
\hline \multicolumn{6}{|l|}{ Mood factors } \\
\hline \multirow[t]{3}{*}{ Alertness } & A & $42 \cdot 7(1 \cdot 4)$ & $42 \cdot 4(1 \cdot 4)$ & $42 \cdot 7(1 \cdot 7)$ & $42 \cdot 3(1 \cdot 2)$ \\
\hline & B & $42 \cdot 2(1 \cdot 2)$ & $42 \cdot 6(1 \cdot 4)$ & $42.5(1.5)$ & $42 \cdot 4(1 \cdot 1)$ \\
\hline & $\mathrm{C}$ & $42 \cdot 7(1 \cdot 6)$ & $42 \cdot 3(3 \cdot 7)$ & $43.5(1.9)$ & $43.3(1.9)$ \\
\hline \multirow[t]{3}{*}{ Contentedness } & $\mathrm{A}$ & $56 \cdot 1(1 \cdot 5)$ & $56 \cdot 3(1 \cdot 5)$ & $56 \cdot 5(1 \cdot 8)$ & $55 \cdot 6(1 \cdot 3)$ \\
\hline & $\mathrm{B}$ & $56 \cdot 1(1 \cdot 5)$ & $56 \cdot 3(1 \cdot 5)$ & $55 \cdot 8(1 \cdot 5)$ & $55 \cdot 9(1 \cdot 2)$ \\
\hline & $\mathrm{C}$ & $56 \cdot 2(1.6)$ & $56.9(1 \cdot 8)$ & $56 \cdot 8(2 \cdot 0)$ & $56 \cdot 6(2 \cdot 0)$ \\
\hline \multirow{3}{*}{ Calmness } & A & $47.5(1 \cdot 8)$ & $47 \cdot 3(1 \cdot 7)$ & $47 \cdot 7(1 \cdot 9)$ & $47 \cdot 0(1 \cdot 6)$ \\
\hline & B & $47 \cdot 4(1 \cdot 8)$ & $47.5(1.7)$ & $47 \cdot 2(1 \cdot 7)$ & $46 \cdot 8(1 \cdot 6)$ \\
\hline & $\mathrm{C}$ & $48 \cdot 0(2 \cdot 1)$ & $48 \cdot 0(1.9)$ & $47 \cdot 8(2 \cdot 0)$ & $47 \cdot 2(1 \cdot 8)$ \\
\hline \multirow[t]{3}{*}{ Anxiety } & $\mathrm{A}$ & $25.6(1.8)$ & $26 \cdot 9(2 \cdot 1)$ & $26 \cdot 4(2 \cdot 0)$ & $21 \cdot 0(1 \cdot 6)$ \\
\hline & B & $22 \cdot 9(2 \cdot 0)$ & $29 \cdot 2(2 \cdot 0)$ & $22.9(1 \cdot 7)$ & $20 \cdot 5(1 \cdot 7)$ \\
\hline & $\mathrm{C}$ & $28 \cdot 6(2 \cdot 2)$ & $22 \cdot 9(1 \cdot 7)$ & $37 \cdot 1(2 \cdot 8)$ & $29 \cdot 3(2 \cdot 9)$ \\
\hline \multirow{3}{*}{ Impaired concentration } & A & $23 \cdot 1(1 \cdot 7)$ & $28 \cdot 7(2 \cdot 2)$ & $23 \cdot 3(1 \cdot 6)$ & $17 \cdot 3(1 \cdot 1)$ \\
\hline & B & $20 \cdot 5(1 \cdot 4)$ & $26 \cdot 6(2 \cdot 0)$ & $19 \cdot 2(1 \cdot 5)$ & $18 \cdot 6(1 \cdot 7)$ \\
\hline & $\mathrm{C}$ & $26 \cdot 7(1 \cdot 9)$ & $22 \cdot 4(1 \cdot 9)$ & $30 \cdot 0(2 \cdot 2)$ & $21 \cdot 4(2 \cdot 1)$ \\
\hline \multirow[t]{3}{*}{ Irritability } & A & $19 \cdot 1(1 \cdot 8)$ & $18 \cdot 7(1 \cdot 5)$ & $18 \cdot 4(1 \cdot 5)$ & $15 \cdot 0(1 \cdot 0)$ \\
\hline & $\mathrm{B}$ & $15 \cdot 4(1 \cdot 1)$ & $18 \cdot 4(1 \cdot 6)$ & $18 \cdot 1(1 \cdot 5)$ & $13 \cdot 8(1 \cdot 2)$ \\
\hline & $\mathrm{C}$ & $23 \cdot 3(1 \cdot 6)$ & $23.8(1.9)$ & $27 \cdot 1(2 \cdot 0)$ & $21.4(1.4)$ \\
\hline \multirow[t]{3}{*}{ Lack of energy } & A & $29 \cdot 6(2 \cdot 1)$ & $33 \cdot 1(2 \cdot 2)$ & $33 \cdot 6(2 \cdot 1)$ & $25 \cdot 7(2 \cdot 0)$ \\
\hline & $\mathrm{B}$ & $33 \cdot 5(2 \cdot 5)$ & $38 \cdot 4(2 \cdot 5)$ & $28 \cdot 6(2 \cdot 2)$ & $27 \cdot 1(2 \cdot 0)$ \\
\hline & $\mathrm{C}$ & $43 \cdot 8(2 \cdot 4)$ & $51 \cdot 4(2 \cdot 6)$ & $41 \cdot 4(2 \cdot 5)$ & $47 \cdot 1(2 \cdot 6)$ \\
\hline \multirow[t]{3}{*}{ Dreams } & A & $40 \cdot 4(2 \cdot 8)$ & $35 \cdot 6(2 \cdot 3)$ & $36.9(2.6)$ & $32 \cdot 7(2 \cdot 3)$ \\
\hline & B & $36 \cdot 3(2 \cdot 9)$ & $46 \cdot 1(2 \cdot 9)$ & $32 \cdot 9(2 \cdot 7)$ & $29 \cdot 1(2 \cdot 3)$ \\
\hline & $\mathrm{C}$ & $33.0(2.5)$ & $32 \cdot 0(2 \cdot 4)$ & $35.5(2 \cdot 9)$ & $37.9(2.6)$ \\
\hline \multirow[t]{3}{*}{ Problems sleeping } & A & $48 \cdot 7(3 \cdot 1)$ & $49 \cdot 8(3 \cdot 0)$ & $40 \cdot 7(2 \cdot 8)$ & $38 \cdot 3(2 \cdot 5)$ \\
\hline & B & $48 \cdot 4(2 \cdot 7)$ & $33 \cdot 7(2 \cdot 3)$ & $37 \cdot 6(2 \cdot 2)$ & $39 \cdot 5(2 \cdot 8)$ \\
\hline & $\mathrm{C}$ & $55 \cdot 0(3 \cdot 7)$ & $42 \cdot 0(3 \cdot 6)$ & $47 \cdot 0(3 \cdot 1)$ & $37 \cdot 1(2 \cdot 8)$ \\
\hline
\end{tabular}

'vitality', group A/B had higher scores than group $\mathrm{C}$ when weeks 0 and 52 were compared $\left(F_{1,55}=6.42, \quad P<0.015\right)$. All these results remained significant when age was covaried. The 'mental health' and 'emotional role' subscales showed a main effect of week that was no longer significant when age was covaried.

\section{Effects of withdrawal on sleep}

\section{Comparison of group $A$ with group $B$}

Sleep diaries completed for a week following each assessment showed no differences between the two withdrawal groups in: (i) time spent in bed (on average, between 8.0 and $8.8 \mathrm{~h}$ across the trial); (ii) number of awakenings per night; or (iii) reported difficulties sleeping.

Visual analogue ratings of 'sleep problems' revealed a significant interaction of group and week $\left(F_{2,80}=3.64, P<0.05\right)$ and a main effect of week $\left(F_{2,80}=3.25, P<0.05\right)$. The interaction remained significant when age was covaried. Group B rated improved sleep between weeks 0 and 12 while they were maintained on their normal dose of sleeping pill while group A's ratings changed very little (Table 4). A parallel effect was seen between weeks 12 and 24: group A rated improved sleep while group B's ratings changed little. At the 52 week follow-up, there was a main effect of week reflecting both groups $\mathrm{A}$ and $\mathrm{B}$ rating fewer sleep problems than they had at week $0\left(F_{1,50}=7 \cdot 34, P<0 \cdot 01\right)$, but this effect was no longer significant when age was covaried.

For dreams, there was an interaction between group and week $\left(F_{2,80}=6.05, P<0.005\right)$ and a main effect of week $\left(F_{2,80}=3.65, P<0.05\right.$; Table 4$)$. When age was covaried, the interaction 
Table 5. Group means (S.D.) on subscales of the SF-36 questionnaire (health-related quality of life) for all patients tested at weeks 0, 12 and 24 (week 52 data are for the subgroup assessed at follow-up)

\begin{tabular}{lccccl}
\hline \hline SF-36 subscale & Group & Baseline & 12 weeks & 24 weeks & $($ Week 52) \\
\hline Physical functioning & A & $62 \cdot 0(25 \cdot 8)$ & $65 \cdot 1(24 \cdot 6)$ & $66 \cdot 5(24 \cdot 7)$ & $58 \cdot 5(27 \cdot 2)$ \\
& B & $64 \cdot 8(26 \cdot 0)$ & $64 \cdot 8(21 \cdot 4)$ & $68 \cdot 4(24 \cdot 1)$ & $58 \cdot 5(20 \cdot 5)$ \\
Social functioning & C & $54 \cdot 3(23 \cdot 3)$ & $55 \cdot 9(24 \cdot 9)$ & $60 \cdot 3(25 \cdot 2)$ & $46 \cdot 8(24 \cdot 5)$ \\
& A & $83 \cdot 1(24 \cdot 1)$ & $88 \cdot 8(20 \cdot 2)$ & $84 \cdot 1(28 \cdot 2)$ & $88 \cdot 4(23 \cdot 6)$ \\
Physical role & B & $92 \cdot 0(15 \cdot 3)$ & $93 \cdot 8(16 \cdot 0)$ & $93 \cdot 4(18 \cdot 3)$ & $96 \cdot 4(16 \cdot 4)$ \\
& C & $78 \cdot 8(24 \cdot 0)$ & $81 \cdot 3(24 \cdot 8)$ & $80 \cdot 0(32 \cdot 0)$ & $71 \cdot 4(38 \cdot 4)$ \\
Emotional role & A & $87 \cdot 6(36 \cdot 9)$ & $78 \cdot 6(38 \cdot 1)$ & $71 \cdot 4(42 \cdot 6)$ & $70 \cdot 7(41 \cdot 2)$ \\
& B & $82 \cdot 2(33 \cdot 3)$ & $79 \cdot 6(37 \cdot 2)$ & $88 \cdot 8(29 \cdot 5)$ & $86 \cdot 9(28 \cdot 1)$ \\
Bodily pain & C & $61 \cdot 3(46 \cdot 9)$ & $56 \cdot 3(49 \cdot 9)$ & $62 \cdot 5(44 \cdot 1)$ & $71 \cdot 4(46 \cdot 9)$ \\
& A & $86 \cdot 5(28 \cdot 6)$ & $86 \cdot 5(32 \cdot 1)$ & $83 \cdot 3(35 \cdot 5)$ & $94 \cdot 3(22 \cdot 0)$ \\
Vitality & B & $90 \cdot 4(26 \cdot 7)$ & $97 \cdot 4(16 \cdot 2)$ & $87 \cdot 7(31 \cdot 4)$ & $92 \cdot 1(25 \cdot 6)$ \\
& C & $73 \cdot 3(38 \cdot 4)$ & $86 \cdot 7(31 \cdot 3)$ & $81 \cdot 7(36 \cdot 6)$ & $90 \cdot 5(27 \cdot 5)$ \\
Mental health & A & $61 \cdot 6(26 \cdot 5)$ & $64 \cdot 1(24 \cdot 2)$ & $63 \cdot 4(28 \cdot 6)$ & $66 \cdot 7(28 \cdot 4)$ \\
& B & $66 \cdot 6(25 \cdot 7)$ & $68 \cdot 3(28 \cdot 7)$ & $66 \cdot 9(23 \cdot 8)$ & $71 \cdot 4(24 \cdot 1)$ \\
& C & $58 \cdot 2(25 \cdot 4)$ & $63 \cdot 6(29 \cdot 9)$ & $65 \cdot 5(22 \cdot 3)$ & $56 \cdot 8(23 \cdot 8)$ \\
& A & $51 \cdot 8(24 \cdot 1)$ & $50 \cdot 4(19 \cdot 8)$ & $54 \cdot 3(21 \cdot 8)$ & $57 \cdot 6(23 \cdot 0)$ \\
& B & $51 \cdot 3(20 \cdot 1)$ & $51 \cdot 8(20 \cdot 3)$ & $65 \cdot 1(20 \cdot 7)$ & $59 \cdot 3(18 \cdot 4)$ \\
& C & $43 \cdot 4(23 \cdot 0)$ & $47 \cdot 5(19 \cdot 1)$ & $45 \cdot 9(20 \cdot 7)$ & $37 \cdot 9(24 \cdot 4)$ \\
& A & $74 \cdot 7(14 \cdot 3)$ & $76 \cdot 7(14 \cdot 1)$ & $78 \cdot 2(22 \cdot 2)$ & $81 \cdot 1(15 \cdot 7)$ \\
\hline \hline
\end{tabular}

remained significant $\left(F_{2,79}=5 \cdot 32, \quad P<0 \cdot 01\right)$. Group B rated more vivid dreams than group A at 12 weeks and less than group A at 24 weeks.

\section{Comparison of withdrawers $(A / B)$ \\ with continuers $(C)$}

There were no significant differences between group $\mathrm{C}$ and the withdrawal groups at weeks 0-24 or at follow-up in ratings of problems sleeping or intensity of dreaming.

\section{Correlations with BZD use}

Correlations were computed between dose of BZD (diazepam equivalent), cumulative dose (years of use $\times$ dose of BZD) and the following measures: change in performance on tests that had shown significant group differences, BZD withdrawal scores and alertness. An $\alpha$ level of 0.01 was adopted to reduce Type I errors.

For the withdrawal groups, dose of BZD correlated positively with ratings of 'problems sleeping' at week $0(r=0.28, P<0.005)$, but there was no correlation at 24 or 52 weeks. For group C, cumulative dose of BZD correlated with ratings of problems sleeping significantly at baseline $(r=0.48, P<0.01)$ and 52 weeks $(r=0.68, P<0.01)$ with a trend towards significance at 24 weeks $(r=0 \cdot 48, P<0 \cdot 04)$. Scores on the BWSQ correlated positively with dose of BZD in the withdrawal groups at week 0 $(r=0.26, \quad P=0.007)$ but not at subsequent assessments. There was no correlation with withdrawal symptoms in group C.

For the withdrawal groups at week 24 only, there was a negative correlation between dose of BZD and the mood factor alertness $(r=-0 \cdot 32$, $P<0.01)$ that reflected increased alertness the higher the previous dose of BZD taken. Irritability ratings in group $\mathrm{C}$ correlated positively with dose of BZD at week $24(r=0.55, P<0 \cdot 01)$ and week $52(r=0 \cdot 79, P<0 \cdot 001)$ with higher irritability scores associated with higher doses. These correlations may relate to sleep problems as irritability ratings correlated with sleep problem ratings at week $24(r=0 \cdot 57, P<0 \cdot 008)$.

\section{DISCUSSION}

In all, $80 \%$ of patients who entered the trial successfully withdrew completely from BZDs by 6 months. This is a high success rate in any longterm BZD using population but especially so in one perceived to be harder to withdraw than younger patients (Holden et al. 1994). A majority of patients had taken BZDs every night for over 10 years and $27 \%$ for more than 
20 years. Withdrawing one group 12 weeks before the second group made little difference to outcome. Differences had been expected on the basis of both Saltzman et al.'s (1992) findings 2-3 weeks after withdrawal and the notion that the acute (or acute-on-chronic) effects of a nightly dose of BZD may influence patients' daytime function. That we found no differences at 12 weeks may reflect both the altered metabolism of BZDs in older people and the length of time required for the brain to adapt following BZD withdrawal.

Our main findings were differences between those who withdrew from BZDs and those who continued to use BZDs. These emerged later than the 12 week assessment point. These results point to several advantages and few disadvantages of older adults withdrawing from BZDs.

\section{What are the advantages for older adults of withdrawal from BZDs?}

At 24 weeks, the sensitive measure of speed of information processing showed that withdrawn groups were performing more accurately than those who continued to use BZDs. Accuracy of information processing improved in those who withdrew compared with a decline in performance by those who continued taking their BZDs. This very significant group difference was found despite all patients performing at very high levels ( $>90 \%$ accuracy). Other group differences in performance emerged later, at 52 weeks.

Reminiscent of Salzman et al.'s (1992) uncontrolled study, patients who withdrew from BZDs had improved working memory at 52 weeks as assessed by total digit span. Again, those who stayed on medication showed a slight decline in performance. Withdrawers also had much improved performance on the second part of the map location test. Indeed, they showed a $22 \%$ improvement at 52 weeks compared with their baseline performance whereas those who stayed on medication showed a small $(5 \%)$ decline. In younger people, chronic use of BZDs was associated with impaired visuospatial ability in a study by Golombok et al. (1988).

Reaction times also improved in withdrawers between 0 and 52 weeks compared with patients who continued medication. This was found not only in the simple task of responding to a single stimulus whenever it appeared on the screen, but also in the more complex speed of information processing task where information had to be held while responding appropriately to the different stimuli. In both these tasks, those who did not wish to withdraw had faster initial reaction times than withdrawers but then declined in speed over the following 12 months. It would seem unlikely that this relative decline reflects normal ageing over this time. It may reflect a relative lack of benefit from practice on the tests, perhaps combined with motivational factors because those who did not withdraw from BZDs had little to gain personally from participating in the study.

Improvements in performance are clearly advantages of withdrawal. Tests such as map search and digit span tap real-life cognitive demands. Most people consult maps to locate places and digit span taps everyday requirements of holding numbers in mind (for example, while dialing the telephone) and of mentally manipulating numbers (for example while calculating what change one is due when shopping). Improved accuracy and speed of information processing would enhance performance in many daily activities, as would faster reaction times generally.

In particular, enhanced information processing, reaction speed and visuospatial abilities would contribute positively to driving performance. Using BZDs has been estimated to approximately double the risk of motor vehicle accidents (Thomas, 1998). Studies in older adults suggest that this increased risk is related to the dose of BZD taken and compounded when alcohol has also been used (Barbone et al. 1998; Hemmelgarn et al. 1997). There is also evidence of an increased risk of falls and fractures in older people who take BZDs (Cummings et al. 1995; Leipzig et al. 1999).

\section{What are the disadvantages for older adults of withdrawal from BZDs?}

There was little evidence of any problems associated with withdrawal. Intriguingly, whether patients were withdrawn earlier or later had no effect on withdrawal symptoms and those who withdrew rated no more BZD withdrawal symptoms than those who stayed on BZDs. Withdrawal symptoms in groups A and B at baseline (but not at 24 or 52 weeks) correlated positively with the dose of BZD taken (i.e. the 
higher the dose, the higher the score on the BWSQ). This suggests that symptoms thought to be associated with withdrawal are dosedependently experienced while patients are taking BZDs.

Although there were some differences in ratings of sleep problems between those who withdrew earlier versus later, these did not reflect increased problems for patients when they withdrew. On the contrary, the findings were that patients rated fewer problems when they were being maintained either on their original dose or on placebo. Sleep diaries showed no differences between the two groups. At no point did the withdrawal groups differ from the group who stayed on medication in terms of sleep ratings. These findings would suggest that when taken over prolonged periods, BZDs do not help people sleep.

Ineffectiveness of BZDs in aiding sleep is also suggested by the significantly positive correlations between measures of BZD intake (dose, cumulative dose) and patients' ratings of sleeping problems. In the withdrawers at baseline, the higher the nightly dose of BZD the greater the rating of sleep problems. At 24 and 52 weeks (i.e. after withdrawal) previous dose did not correlate with sleep problems. However, in those who stayed on medication, there was a positive correlation at all assessment points showing higher cumulative intake of BZDs was associated with increased sleep problems.

There was no evidence of emergent depression or anxiety on withdrawing from BZDs. Indeed the only group to show elevated anxiety ratings during the trial was $\mathrm{C}$ (at 24 weeks), i.e. those patients who had stayed on medication. Few changes were obtained on any other measures of mood. Interestingly, alertness ratings by withdrawers at week 24 were significantly associated with dose of BZD previously taken reflecting an increased alertness postwithdrawal. Patients often remarked on their increased feeling of alertness. 'I feel sharper when I wake up'; 'I feel better, more awake'; 'It used to take me an hour to fully wake up.'

\section{Should clinicians continue to prescribe BZDs on a long-term basis to older adults?}

This was the question initially posed by the study. The answer is that there are some subtle cognitive advantages to withdrawal and negligible costs in terms of discomfort to patients. In terms of service costs, success rates in withdrawal would be maximized if patients were provided with: (i) a tapered dose regime (preferably down to placebo capsules); (ii) information about sleep; and (iii) psychological support. There will also be potential savings to the service in terms of reduced drug costs, and possibly through fewer road traffic accidents and fewer falls and fractures. In terms of health economics, although the costs of BZD medication is fairly small, there are very substantial costs in treating fractures and other injuries associated with accidents and falls.

More than half $(57 \%)$ of the patients initially interviewed wanted to take part in the withdrawal programme. The $43 \%$ who did not wish to withdraw could foresee no advantage to withdrawal and felt that their BZDs were effective in helping them sleep. Our present findings of cognitive advantages to withdrawal and lack of effectiveness of long-term BZDs in helping sleep may influence such patients in deciding whether or not to stop taking BZDs. For some older people, the promise of improved cognitive function with little discomfort may be sufficient to persuade them to stop BZDs.

The majority of patients who were taking temazepam $10 \mathrm{mg}$ were withdrawn over 6 weeks and those on $5 \mathrm{mg}$ nitrazepam or $1 \mathrm{mg}$ loprazolam were withdrawn over 4 weeks. The same tapering doses as used in this study could be made up for patients in primary care. Practice nurses might be trained to support older adults in withdrawing from BZDs. As in the present study, patients on repeat prescriptions of BZDs could be invited to discuss their sleep and the advantages of BZD withdrawal. Numbered bottles containing the appropriate doses could be given monthly to those who wish to withdraw and the nurse could provide support and education about sleep. It would be important to provide tapering doses down to placebo and maintain patients on placebo for several weeks. Clinically, in terms of withdrawal anxieties, the main concern of patients in the study was at week 24 when their capsules were stopped and it was important that we could reassure them that they had taken only an inactive placebo for several weeks before this point. 


\section{Methodological considerations}

Patients were not randomly allocated to group $\mathrm{C}$ as it was not considered ethical to keep people who wished to withdraw from BZDs on the drugs for a prolonged period. Non-random allocation introduces a selection bias into trials. Despite this, the patients in this group were well matched with those in the other two groups on all demographic variables: age, gender, premorbid function, BZD used, dose and duration of BZD use. Further, all the groups performed similarly at baseline.

Patient numbers are inevitably a consideration in longitudinal studies and although these were acceptable in those who withdrew from BZDs (with over 50 patients at week 52) there was a higher proportional drop out rate in patients who stayed on BZDs with just 15 being assessed at week 52 . This probably reflects the fact that patients not withdrawing from BZDs had nothing to gain personally from taking part in the research.

\section{Implications for future research}

Our present findings add to the debate on cognitive recovery following chronic BZD use in showing that some aspects of working memory, attention and visuospatial processing do show improvement between 6 and 10 months after BZD cessation. Although working memory improved in those who withdrew from BZDs, we did not find an improvement in episodic memory (tapped by prose recall). Episodic memory is the memory system most sensitive to decline with age and with dementia. It is also highly sensitive to impairment by BZDs. There is evidence suggesting that although tolerance builds up to the sedative and attentional effects of BZDs, tolerance does not fully develop to their amnestic effects. For example, in younger, long-term BZD users, a challenge dose of $10 \mathrm{mg}$ diazepam produces impairments on tasks tapping episodic memory but not tasks tapping only attentional function or sedation (Gorenstein et al. 1994). The only randomized, placebo controlled trial of the cognitive effects of BZDs in anxious patients showed that impairments in memory persisted several weeks beyond withdrawal (Curran et al. 1994) but were no longer evident 3.5 years later (Kiliç et al. 1999). Tata et al. (1994) found little improvement in episodic memory function 6 months after 21 patients had withdrawn from high (mean $42 \mathrm{mg}$ diazepam) doses. Thus, there may be a differential profile of recovery following BZD withdrawal on episodic memory, working memory and other cognitive processes. It may be that episodic memory improves following BZD withdrawal but at a later point in time. It would be important to follow-up withdrawers from this study to determine whether or not this is the case.

All the patients in the present study were long-term users of BZDs and it would be helpful to determine whether chronic use produces changes in cognitive ageing. There is animal evidence that BZDs/GABA are implicated in the normal process of brain and cognitive ageing (Marczynski et al. 1994). It would therefore be important to assess a group of older adults who had never taken BZDs but could be matched for age and pre-morbid intellectual function with patients in this study. We have recently begun to assess this group.

As prescriptions for BZDs have decreased, there has been a parallel increase in prescriptions of 'newer' hypnotics such as zopiclone or zolpidem. Although classified as "nonbenzodiazepines', these drugs have very similar properties to BZDs. For example, an acute dose of zopiclone can impair memory and psychomotor function (e.g. Mattila et al. 1998). Although it is recommended that prescriptions for these drugs should be limited to 4 weeks, in practice this is not always the case and it is important that repeat prescribing is monitored.

In terms of clinical practice, the findings of the present study suggest that withdrawal may be aided by the patient being unsure of the timing of when their dose of drug is reduced to zero i.e. a placebo. This 'blinded tapering therapy' (BTT) seems an effective approach to long-term use of BZDs and there are clear theoretical reasons why this should be so. BTT essentially separates out two major aspects of drug taking: psychological and pharmacological. We found little in the way of withdrawal problems or sleep problems as drug dose was tapered (i.e. during pharmacological change). However, when placebo was stopped abruptly at 24 weeks, psychological aspects of drug taking (i.e. those associated with the behaviour of taking the capsule before bed and cognitions about the effectiveness of the capsule in aiding sleep) 
affected many patients who were then re-assured that they had taken placebo for the previous few weeks. In terms of clinical practice, there are ethical considerations of BTT in using placebos and blind tapering. However, its potential efficacy would mean it could be considered for some patients who take centrally acting drugs for extended periods of time (for example, those using BZD anxiolytics or people on methadone maintenance). Research on this approach to treatment would be clearly warranted and may ultimately have important implications for treatment of long-term drug users, improving their health and reducing service costs.

This study was funded by a grant to H.V.C., S.I. and B. W. by the NHS Executive London (NHSE-LRO), Research and Development, Responsive Funding Programme. We are very grateful to all the patients and GPs who took part in this project and to practice staff whose help was invaluable. Sharon See Tai kindly contributed to statistical analyses. We would also like to thank Tony Roth for helpful discussion, Keith Wesnes for the speed of information processing task and Amanda Burgess and Barbara Salmon at NHSE whose help ensured the project's successful completion.

\section{REFERENCES}

Andresen, E. M., Patrick, D. L., Carter, W. B. \& Malmgren, J. A. (1995). Comparing the performance of health status measures for healthy older adults. Journal of the American Geriatric Society 43, 1030-1034.

Baddeley, A., Emslie, H. \& Nimmo-Smith, I. (1992). The Speed and Capacity of Language Processing. Thames Valley Test Company: Reading.

Barbone, F., McMahon, A. D., Davey, P. G., Morris, A. D., Reid, I. C., McDevitt, D. G. \& MacDonald, T. M. (1998). Association of road-traffic accidents with benzodiazepine use. Lancet $\mathbf{3 5 2}$, 1331-1336.

Barbui, C., Gregis, M. \& Zappa, M. (1998). A cross-sectional audit of benzodiazepine use among general practice patients. Acta Psychiatrica Scandinavica 97, 153-156.

Bond, A. J. \& Lader, M. H. (1974). The use of analogue scales in rating subjective feelings. British Journal of Medical Psychology 47, 211-218.

Bowling, A. (1991). Measuring Health: A Review of Quality of Life Measurement Scales. Open University Press: Buckingham.

Campbell, A. (1991). Drug treatment as a cause of falls in old age. Drugs and Aging 1, 289-302.

Colenda, C. C., Mickus, M. A., Marcus, S. C., Tanielian, T. L. \& Pincus, H. A. (2002). Comparison of adult and geriatric psychiatric practice patterns: findings from the American Psychiatric Association's Practice Research Network. American Journal of Geriatric Psychiatry 10, 609-617.

Cummings, S. R., Nevitt, M. C., Browner, W. S., Stone, K., Fox, K. M., Ensrud, K. E., Cauley, J., Black, D. \& Vogt, T. M. (1995). Risk factors for hip fracture in white women. New England Journal of Medicine 332, 767-773.
Curran, H. V. (1991). Benzodiazepines, memory and mood: a review. Psychopharmacology 105, 1-8.

Curran, H. V. (2000). The psychopharmacology of memory. In The Oxford Handbook of Memory (ed. E. Tulving and F. Craik), pp. 539-556. Oxford University Press: New York.

Curran, H. V., Bond, A., O'Sullivan, G., Bruce, M., Marks, I., Lelliot, P., Shine, P. \& Lader, M. (1994). Memory functions, alprazolam and exposure therapy: a controlled longitudinal trial of agoraphobia with panic disorder. Psychological Medicine $\mathbf{2 4}$ 969-976.

D'Ath, P., Katona, P., Mullan, E., Evans, S. \& Katona, C. (1994) Screening, detection and management of depression in elderly primary care attenders: the acceptability and performance of the 15 item geriatric Depression Scale (GDS15) and the development of short versions. Family Practice 11, 260-266.

Egan, M., Moride, Y., Wolfson, C. \& Monette, J. (2000). Long-term continous use of benzodiazepines by older adults in Quebec: prevalence, incidence and risk factors. Journal of the American Geriatric Society 48, 811-816.

Golombok, S., Moodley, P. \& Lader, M. (1988). Cognitive impairment in long-term benzodiazepines users. Psychological Medicine 18, 365-374.

Gorenstein, C., Bernik, M. A. \& Pompeia, S. (1994). Differential acute psychomotor and cognitive effects of diazepam on long-term benzodiazepine users. International Clinical Psychopharmacology 9, 145-153.

Gray, S. L., Lai, K. V. \& Larson, E. B. (1999). Drug-induced cognition disorders in the elderly: incidence, prevention and management. Drug Safety 21, 101-122.

Greenblatt, D., Harmatz, J. S., Shapiro, L., Engelhardt, N., Gouthro, T. A. \& Shader, R. I. (1991). Sensitivity to triazolam in the elderly. New England Journal of Medicine 324, $1326-1331$.

Griffiths, R. R. (1995). Commentary - Benzodiazepines: long-term use among patients is a concern. Psychopharmacology 118, 116-117.

Hayes, V., Morris, J., Wolfe, C. \& Morgan, M. (1995). The SF-36 Health Survey Questionnaire: is it suitable for older adults? Age and Ageing 24, 120-125.

Hinrichs, S. \& Ghoneim, M. M. (1987). Diazepam, behavior and ageing. Psychopharmacology 92, 100-105.

Hemmelgarn, B., Suissa, S., Huang, A., Boivin, J. F. \& Pinard, G. (1997). Benzodiazepine use and the risk of motor vehicle crash in the elderly. Journal of the American Medical Association 278, $27-31$.

Holden, J. D., Hughes, I. M. \& Tree, A. (1994). Benzodiazepine prescribing and withdrawal for 3234 patients in 15 general practices. Family Practice 11, 358-362.

Iliffe, S., Curran, H. V., Collins, R., Kee, S. C. H., Fletcher, S. \& Woods, B. (2003). Attitudes to long-term use of benzodiazepine hypnotics by older people in general practice: findings from interviews with service users and providers. Ageing and Mental Health (in the press).

Jorm, A. F., Grayson, D., Creasey, H., Waite, L. \& Broe, G. A. (2000). Long-term benzodiazepine use by elderly people living in the community. Australian and New Zealand Journal of Public Health 24, 7-10.

Kiliç, C., Curran, H. V., Noshirvani, H., Marks, I. M. \& Başoğlu, M. (1999). Long-term effects of alprazolam on memory: a 3.5 year follow-up of agoraphobial panic patients. Psychological Medicine 29, 225-231.

Lader, M. H. (1992). The Medical Management of Insomnia in General Practice. Royal Society of Medicine Round Table Series, 28. Royal Society of Medicine: London.

Leipzig, R. M., Cumming, R. G. \& Tinetti, M. E. (1999). Drugs and falls in older people: a systematic review and meta-analysis: I. Psychotropic drugs. Journal of the American Geriatric Society 47 30-39.

Lyons, R., Perry, H. M. \& Littlepage, B. N. (1994). Evidence for the validity of the short-form 36 questionnaire (SF-36) in an elderly population. Age and Aging 23, 182-184. 
Marczynski, T. J. (1995). GABAergic deafferentation hypothesis of brain aging and Alzheimer's disease; pharmacologic profile of the benzodiazepine antagonist, flumazenil. Review of Neuroscience $\mathbf{6}$, 221-258.

Mattila, M. J., Vanakoski, J., Kalska, H. \& Seppala, T. (1998). Effects of alcohol, zolpidem, and some other sedatives and hypnotics on human performance and memory. Pharmacology, Biochemistry and Behaviour 59, 917-923.

Morgan, K. (1994). Hypnotic drugs, psychomotor performance and ageing. Journal of Sleep Reviews 3, 1-15.

Morgan, K. \& Clarke, J. (1995). Longitudinal Trends in Late-Life Insomnia. Sleep Disorders Assessment and Treatment: Proceedings of the British Sleep Society Conference. St Georges Hospital Mental Health Science Conference Unit: London.

Morgan, K. \& Gledhill, K. (1991). Managing Sleep and Insomnia in the Older Person. Winslow Press: Bicester.

Nikaido, A. M., Ellinwood, E. H., Heatherly, D. G. \& Gupta, S. K. (1990). Age-related increase in CNS sensitivity to benzodiazepines as assessed by task difficulty. Psychopharmacology 100, 90-97.

Pomara, N., Stanley, B., Block, R., Berchou, R. C., Stanley, B., Greenblatt, D. J., Newton, R. E. \& Gershon, S. (1985). Increased sensitivity of the elderly to the effects of diazepam. Journal of Clinical Psychiatry 46, 185-187.

Robertson, I. H., Ward, T., Ridgeway, V. \& Nimmo-Smith, I. (1996). The structure of normal human attention: the Test of Everyday Attention. Journal of the International Neuropsychological Society 6, 525-534.

Rumble, R. H. \& Morgan, K. (1994). Longitudinal trends in prescribing for elderly patients: two surveys four years apart. British Journal of General Practice 44, 571-575.
Salzman, C., Fisher, J., Nobel, K., Glassman, R., Wolfson, A. \& Kelley, M. (1992). Cognitive improvement following benzodiazepine discontinuation in elderly nursing home residents. International Journal of Geriatric Psychiatry 7, 89-93.

Shorr, R. \& Robin, D. (1994). Rational use of benzodiazepines in the elderly. Drugs and Aging 4, 9-20.

Tata, P. R., Rollings, J., Collins, M., Pickering, A. \& Jacobson, R. R. (1994). Lack of cognitive recovery following withdrawal from long-term benzodiazepine use. Psychological Medicine 24, 203-213.

Thomas, R. E. (1998). Benzodiazepine use and motor vehicle accidents. Systematic review of reported association. Canadian Family Physician 44, 799-808.

Ticehurst, S. (1995). Alcohol and drug abuse. In Neurotic Disorders in the Elderly (ed. J. Linsey), pp. 56-71. Oxford University Press: Oxford.

Tyrer, P., Murphy, S. \& Riley, P. (1990). The Benzodiazepine Withdrawal Symptoms Questionnaire. Journal of Affective Disorders 19, 53-61.

Wechsler, D. (1955). Manual for the Wechsler Adult Intelligence Scale. Psychological Corporation: New York.

Wesnes, K. A., Ward, T., McGinty, A. \& Petrini, O. (2000). The memory enhancing effects of a Ginkgo biloba/Panax ginseng combination in healthy middle aged volunteers. Psychopharmacology 152, 353-361.

Wilson, B., Cockburn, J. \& Baddeley, A. (1985). The Rivermead Behavioural Memory Test. Thames Valley Test Company: Reading.

Woods, J. H. \& Winger, G. (1995). Current benzodiazepine issues. Psychopharmacology 118, 107-115.

Woods, J. H., Katz, J. L. \& Winger, G. (1992). Benzodiazepine use, abuse and consequences. Pharmacology Review 44, 151-347. 